\title{
Erratum to: Hierarchically porous carbon foams for electric double layer capacitors
}

Feng Zhang ${ }^{1,2, \S}$, Tianyu Liu ${ }^{2, \S}$, Guihua Hou ${ }^{1}$, Tianyi Kou ${ }^{2}$, Lu Yue ${ }^{1}$, Rongfeng Guan ${ }^{1}$, and Yat $\mathrm{Li}^{2}(\varangle)$

${ }^{1}$ Key Laboratory for Advanced Technology in Environmental Protection of Jiangsu Province, Yancheng Institute of Technology, Yancheng 224051, China

${ }^{2}$ Department of Chemistry and Biochemistry, University of California, Santa Cruz, 1156 High Street, Santa Cruz, CA 95064, USA

${ }^{\S}$ These authors contributed equally to this work.

(C) Tsinghua University Press and Springer-Verlag Berlin Heidelberg 2016

\section{Erratum to}

Nano Research

DOI 10.1007/s12274-016-1173-z

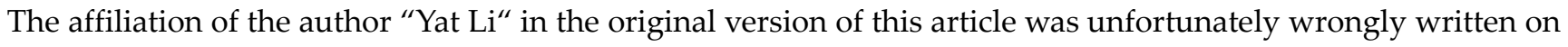
the first pages of the main text and the ESM, instead of

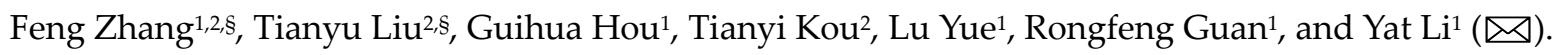

It should read

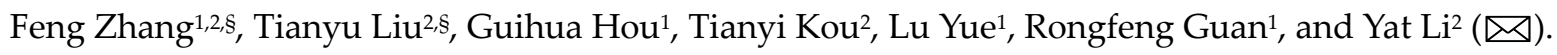

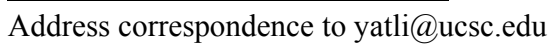

